\title{
PERFIL E INTENSIDADE DE SINTOMAS DE MULHERES NO CLIMATÉRIO AVALIADAS EM UNIDADES BÁSICAS DE SAÚDE DE PRESIDENTE PRUDENTE
}

Lara Nery Peixoto, Mariane Fatima da Silva Araujo, Carolina Andrade Egydio, Fernanda Elisa Ribeiro, Cristina Elena Prado Teles Fregonesi, Edna Maria do Carmo

Universidade Estadual Paulista - FCT/UNESP. Pós-Graduação Lato Sensu em Fisioterapia, Presidente Prudente, SP. email: larinha-peixoto@hotmail.com

\section{RESUMO}

O climatério é um processo de mudanças físicas e emocionais, influenciado por múltiplos fatores, cujo conhecimento é fundamental para uma assistência mais qualificada. O objetivo foi verificar o perfil e intensidade dos sintomas de mulheres no climatério em Unidades Básicas de Saúde de Presidente Prudente. Foram avaliadas 56 mulheres com idade entre 40 e 65 anos, com entrevista investigando características sociodemográficas, ginecológicas e intensidade de sintomas climatéricos pelo Índice Menopausal de Blatt-Kupperman. A média de idade foi de 51,1 $\pm 5,47$ anos, $53,6 \%$ eram brancas, $71,4 \%$ eram casadas, $62,5 \%$ estudaram até o ensino fundamental e $75 \%$ não trabalhavam. Também observou-se que $25 \%$ realizaram histerectomia, $60,7 \%$ apresentava incontinência urinária e $55,4 \%$ possuíam vida sexual ativa. Quanto aos sintomas, $21,4 \%$ apresentaram intensidade grave, $46,4 \%$ moderado e $30,4 \%$ leve. Conhecer os fatores envolvidos no climatério é determinante para os profissionais da saúde promover um cuidado mais integral e humanizado para essa população.

Palavras-chave: Saúde da mulher, Climatério, Menopausa, incontinência urinária, Sintomas.

\section{PROFILE AND INTENSITY OF SYMPTOMS OF WOMEN IN CLIMACTERIC EVALUATED IN HEALTH BASIC UNITS OF PRESIDENTE PRUDENTE}

\begin{abstract}
Menopause is a process of physical and emotional changes, influenced by multiple factors, which is essential for a more qualified assistance. The goal was to determine the profile and intensity of symptoms in menopausal women in Basic Health Units in Presidente Prudente. 56 women aged between 40 and 65 years, investigating interview with sociodemographic, gynecological characteristics and intensity of climacteric symptoms by menopausal index of Kupperman-Blatt were evaluated. The average age was $51.1 \pm 5.47$ years, $53.6 \%$ were white, $71.4 \%$ were married, $62.5 \%$ had completed elementary school and $75 \%$ did not work. Also it was observed that $25 \%$ underwent hysterectomy, $60.7 \%$ had urinary incontinence and $55.4 \%$ had active sex life. As for symptoms, $21.4 \%$ had severe intensity, $46.4 \%$ moderate and $30.4 \%$ mild. Knowing the factors involved in menopause is crucial for health professionals to promote a more comprehensive and humane care for this population.
\end{abstract}

Keywords: Women's Health, Climacterium, Menopause, Urinary Incontinence, Symptoms. 


\section{INTRODUÇÃO}

O envelhecimento da população mundial é fenômeno recente na história da humanidade. O Brasil, com as características de um país em desenvolvimento, passa pelo processo de aumento da expectativa de vida. A expectativa de vida da mulher é de 72,5 anos, com um aumento significativo das mulheres acima de 45 anos, a quem são oferecidas uma inadequada atenção à saúde durante o climatério, levando a frequentes queixas nas consultas de atenção primária a saúde e percepção de queda na qualidade de vida desta população ${ }^{1}$.

O climatério é definido pela Organização Mundial da Saúde (OMS) como uma fase biológica da vida e não um processo patológico, que compreende o final do período reprodutivo e o início da senilidade, marcado por eventos importantes como a menopausa, somente reconhecido depois de passados 12 meses da última menstruação e acontece geralmente em torno dos 50 anos de idade 2 .

Durante o período climatérico, cerca de 60 a $80 \%$ das mulheres refere algum tipo de sintomatologia com sua maioria atribuída ao estado de hipoestrogenismo, que ocorre pelo declínio da função ovariana. Os sinais e sintomas mais comuns são irregularidade menstrual, aparecimento ou agravamento do quadro de tensão pré-menstrual e cólica menstrual, palpitações, tonteiras, cansaço, memória fraca, cefaléia, dores articulares, ansiedade, irritabilidade, insônia, depressão, dispaurenia, urgência miccional, cistite, incontinência urinária, secura vaginal e os "fogachos" ou onda de calor ${ }^{3-5}$. Além disso, o estado de hipoestrogenismo pode interferir na sexualidade feminina, juntamente com a sintomatologia, mudanças da configuração corporal, e fatores psicológicos, sociais e culturais ${ }^{6}$.

Acrescenta-se ainda que a frequência e a intensidade com que a sintomatologia aparece tem ampla margem de variação e pode estar intimamente associada a alterações na qualidade de vida feminina no que se refere a aspectos como o bem estar físico, social, espiritual e emocional. Portanto, o climatério é caracterizado como um processo de profundas mudanças físicas e emocionais, influenciado por múltiplos fatores inerentes ao psiquismo, à cultura, ao ambiente, a história de vida pessoal, dentre outros $^{7,8}$.

O estímulo à adoção de hábitos saudáveis que possam envolver uma melhoria da qualidade de vida e promover o alívio da sintomatologia climatérica inclui a mudança quanto aos hábitos alimentares, prática regular de atividade física, prescrição adequada de fármacos para redução de sintomas desagradáveis, tais como fogachos 
e secura vaginal, estímulo a atividades grupais de lazer e recreação, bem como apoio psicológico para a elevação da autoestima e alcance do bem-estar ${ }^{8-11}$.

Outro dado relevante é que esta população recebe informação insuficiente sobre esta fase, demonstrando que há uma deficiência de informação nos serviços de saúde que prestam atendimento a saúde da mulher ${ }^{6}$.

Sendo assim, esse estudo teve como objetivo avaliar o perfil e intensidade de sintomas de mulheres no climatério, visando conhecer e contribuir para a solução dos problemas delas e, ao mesmo tempo, retratar as características quanto às situações vivenciadas neste período.

\section{METODOLOGIA}

Trata-se de um estudo transversal descritivo onde participaram 56 mulheres com idade entre 40 e 65 anos, atendidas em Unidades Básicas de Saúde da cidade de Presidente Prudente. As participantes foram devidamente informadas sobre os procedimentos e objetivos deste estudo e aquelas que concordaram, assinaram o "Termo de Consentimento Livre e Esclarecido". Esse estudo faz parte do projeto de pesquisa "Percepção da saúde de mulheres no climatério em Unidades Básicas de Saúde de Presidente Prudente", aprovado no

protocolo

CAAE

$n \underline{0}$

24190213.5.0000.5402.

Para coleta de dados foi realizada uma entrevista semiestruturada com dados sociodemográficos, como idade, raça, estado civil, grau de instrução, ocupação, renda familiar e dados ginecológicos, do estilo de vida e sintomas climatéricos.

Para verificar a intensidade dos sintomas climatéricos utilizou-se o Índice Menopausal de Blatt e Kupperman (IMBK). No IMBK, os diversos sintomas incluídos sob a denominação de síndrome climatérica (fogachos, insônia, parestesia, nervosismo, melancolia, vertigem, artralgia/mialgia, cefaleia, palpitação e zumbido) recebem valores numéricos de acordo com a sua intensidade (leves=1, moderados $=2$, severos $=3$ ) multiplicados pelos fatores de conversão preconizados por Kupperman, que representam a importância do sintoma na síndrome climatérica. Desta forma, os sintomas vasomotores podem receber valores 4, 8 ou 12; a parestesia, a insônia e o nervosismo, 2, 4 ou 6; e o restante (melancolia, vertigem, artralgia/mialgia, cefaleia, palpitação e zumbido), os valores 1 , 2 ou 3. O escore global da soma desses valores é catalogado em intensidade leve, se somatório dos valores até 19; moderado, se entre 20 e 35 ; e severa, se maior que $35^{12}$. 
As características da amostra e a análise dos resultados foram realizadas por estatística descritiva, onde os valores foram descritos como absolutos.

\section{RESULTADOS}

A amostra foi composta de 56 mulheres, com média de idade de 51,1 $\pm 5,47$ anos. Os resultados seguem apresentados nas tabelas 1, 2 e 3, mostrando perfil sociodemográfico, ginecológico, estilo de vida e sintomas climatéricos.

Tabela 1. Perfil sociodemográfico.

\begin{tabular}{l|cc}
\hline \multicolumn{1}{c|}{ Estado Civil } & Frequência & Percentual \\
Casada & 40 & 71,4 \\
Solteira & 3 & 5,4 \\
Amasiada & 4 & 7,1 \\
Divorciada & 6 & 10,7 \\
Viúva & 3 & 5,4 \\
$\quad$ Raça & 30 & 53,6 \\
Branca & 10 & 17,9 \\
Negra & 14 & 25,0 \\
Parda & 2 & 3,6 \\
Amarela & & \\
\multicolumn{1}{c}{ Grau de Instrução } & 3 & 5,4 \\
Ensino Superior & 14 & 25,0 \\
Ensino Médio Completo & 4 & 7,1 \\
Ensino Médio Incompleto & 8 & 14,3 \\
Ensino fundamental Completo & 27 & 48,2 \\
Ensino Fundamental Incompleto & & \\
$\quad$ Ocupação & 14 & 25,0 \\
Trabalha & 42 & 75,0 \\
Não trabalha & & \\
$\quad$ Renda Familiar & 12 & 21,4 \\
Até 1 salário mínimo & 36 & 64,3 \\
2 a 3 salários mínimos & 8 & 14,3 \\
Mais que 4 salários mínimos & & \\
\hline
\end{tabular}


Tabela 2. Perfil ginecológico, atividade física e sintomas climatéricos quanto à intensidade.

\begin{tabular}{c|cc}
\hline $\begin{array}{c}\text { Reposição Hormonal } \\
\text { Sim }\end{array}$ & Frequência & Percentual \\
Não & 45 & 19,6 \\
Menstruação & & 80,4 \\
Sim & 23 & 41,1 \\
Não & 33 & 58,9 \\
Vida sexual ativa & & \\
Sim & 31 & 55,4 \\
Não & 25 & 44,6 \\
Incontinência Urinária & & \\
Sim & 34 & 55,4 \\
Não & 22 & 44,6 \\
Atividade Física & & \\
Sim & 45 & 80,4 \\
Não & 11 & 19,6 \\
Sintomas Climatéricos & & \\
Nenhum & 1 & 1,8 \\
Leve & 17 & 30,4 \\
Moderado & 26 & 46,4 \\
Grave & 12 & 21,4 \\
\hline
\end{tabular}

É necessário ressaltar que dentre as mulheres que relataram vida sexual ativa ( $n=31), 48,4 \%$ tinha dor durante a relação sexual, e das 45 mulheres que relataram praticar atividade física, $25(55,6 \%)$ realizam alguma atividade 3 vezes por semana ou mais.

\section{DISCUSSÃO}

É reconhecido que há uma variedade de fatores que envolvem a fase do climatério, bem como o quanto ele pode influenciar a qualidade de vida da mulher que o vive, sendo de extrema importância que esses fatores sejam conhecidos e considerados para que os profissionais da saúde possam atender às necessidades dessa mulher, com o propósito de lhe proporcionar uma assistência globalizada, incluindo a assistência às suas necessidades biopsicossociais, como preconiza o Ministério da Saúde ${ }^{13}$.

As 56 mulheres estudadas apresentaram idade variando de 40 a 65 anos, com média de 51,1 anos e $71,4 \%$ eram casadas, o que está de acordo com outros estudos $^{6-9,14}$, que avaliaram que a faixa etária do climatério ou menopausa era de 40 a 65 anos, sendo a maioria casada. Em relação à escolaridade $62,5 \%$ delas estudaram até o ensino fundamental, quanto à ocupação, $75 \%$ não trabalham fora, e a renda familiar da 
maioria $(85,7 \%)$ das mulheres avaliadas no presente estudo era de até 3 salários mínimos, sendo que $21,4 \%$ sobreviviam com renda familiar de 1 salário apenas, o que também foi observado no estudo de Silva Filho, onde a profissão predominante foi a de donas de casa, o nível de escolaridade é ensino fundamental incompleto e a renda familiar da maioria era de até $\mathrm{R} \$ 1.343,00^{14}$.

Das entrevistadas, apenas 19,6\% fazem terapia de reposição hormonal, o que poderia justificar $\mathrm{o}$ alto índice de sintomas climatéricos nessa população, pois segundo Pines et al. a terapia hormonal continua sendo o tratamento mais eficaz para sintomas vasomotores e urogenitais decorrentes do hipoestrogenismo ${ }^{15}$.

Quanto ao fato de $41,1 \%$ das entrevistadas apresentarem menstruação, e ainda assim relatarem sintomas climatéricos, pode ser que algumas têm o sistema termorregulador menos estável ou são mais sensíveis a essas mudanças e, portanto, são mais susceptíveis a experimentar sintomas no climatério, como foi visto no estudo de Pedro et $\mathrm{al}^{16}$.

No presente estudo constatou-se que 44,6\% das mulheres não apresentam vida sexual ativa, e dentre as mulheres que relataram vida sexual ativa $(n=31), 48,4 \%$ tinha dor ou incômodo durante a relação sexual. Segundo Teles et al. o que caracteriza o período do climatério são os transtornos sexuais, pois estão relacionados ao hipoestrogenismo e às alterações dos níveis de testosterona. A dificuldade sexual pode ser atribuída à atrofia vaginal caracterizada pelo afinamento do epitélio, perda da rugosidade e redução da secreção vaginal, as quais podem levar a mulher climatérica a ter problemas conjugais ${ }^{6}$.

Vários estudos com mulheres no período do climatério têm explorado a relação dessa fase com a incontinência urinária (IU), como o realizado por Pedro et al. $^{16}$ com 456 mulheres no período de climatério, que evidenciou uma prevalência de $27,4 \%$ de IU nesse grupo. Outro estudo que investigou esse fato foi o de Coelho et al. ${ }^{17}$, que entrevistou 207 mulheres, a partir de 18 anos, onde a prevalência de incontinência foi de $73,5 \%$ nas mulheres na faixa etária de 48 a 57 anos e de $76 \%$ para mulheres com idade acima de 58 anos. No presente estudo foi verificada uma prevalência de $55,4 \%$ de mulheres que apresentam IU.

De acordo com a prática de atividade física, $80,4 \%$ das entrevistadas relataram praticar algum tipo de atividade física, mas apenas $55,6 \%$ realizam alguma atividade três vezes por semana ou mais. Segundo o estudo de De Lorenzi et al. a sintomatologia climatérica mostrou-se significantemente mais intensa entre as mulheres que não praticavam atividade física regular ${ }^{4}$ o 
exercício físico estimula a secreção de endorfinas hipotalâmicas, substâncias envolvidas na termorregulação hipotalâmica, reduzindo os sintomas vasomotores, presentes nesta fase do climatério $^{18}$. Além disso, o combate ao sedentarismo, por meio de exercícios físicos regulares, melhora a aptidão física, favorece a disposição para viver, além de combater doenças e agravos à saúde, tais como: obesidade, osteoporose, doenças cardiovasculares, ansiedade, depressão e artralgias, um dos sintomas mais frequentes no climatério ${ }^{19}$.

Quanto aos sintomas climatéricos, apenas uma entrevistada não relatou sintomas climatéricos, 30,4\% apresentaram sintomas leves, $46,4 \%$ moderados e $21,4 \%$ sintomas acentuados, o que está de acordo com os resultados do estudo de Pedro et al. ${ }^{16}$, onde a sintomatologia associada à síndrome do climatério foi altamente prevalente, sendo que cerca de $96,9 \%$ das mulheres experimentaram pelo menos um dos sintomas. Santos et al. ${ }^{20}$ em seu estudo observou que, de acordo com o índice de Kupperman, 26,8\% das pacientes apresentaram sintomatologia leve, 60,9\% moderada e $12,2 \%$ acentuada. 0 estudo apontou que as mulheres entrevistadas apresentaram sintomas muitas vezes iguais, mas foi percebido que cada uma pode apresentar particularidade diante das manifestações.
Portanto, uma maior atenção deve ser dada a esse período para que os sintomas climatéricos sejam minimizados por meio de orientações como a realização de atividades físicas e alimentação saudável. Para que essas ações sejam eficazes é necessário que os profissionais de saúde conheçam as características gerais dessa população, mas levando em consideração que cada mulher pode apresentar particularidades diante das alterações desse período.

\section{CONCLUSÃO}

Este estudo mostrou que as mulheres atendidas em Unidades Básicas de Saúde de Presidente Prudente apresentam em sua maioria algum sintoma climatérico, sendo que a maior porcentagem delas apresentam esses sintomas com intensidade moderada. Visto que esses sintomas podem interferir na qualidade de vida da mulher, conhecer esses dados juntamente com as características dessa população se faz extremamente necessário, contribuindo assim para a solução dos problemas que aparecem nessa fase, promovendo um cuidado mais integral e humanizado para essa população.

\section{REFERÊNCIAS}

1. De Lorenzi DRS, Catani LB, Moreira K, Ártico GR. Assistência à mulher climatérica: novos paradigmas. Rev. Bras. Enferm, Brasília. V.62, n.2, p.287-93, mar-abril, 2009. 
2. OMS - Organização Mundial de Saúde. Série de Informes Técnicos. Investigação sobre a menopausa nos anos noventa. Genebra; 1996.

3. Baracho, E. Fisioterapia aplicada à saúde da mulher. 5a ed. Rio de Janeiro: Guanabara Koogan, 2012.

4. De Lorenzi DRS, Danelon C, Saciloto B. Fatores indicadores da sintomatologia climatérica. Rev Bras Ginecol Obstet. 27(1):12-9, 2005. http://dx.doi.org/10.1590/S0100$\underline{72032005000100004}$

5. Nelson HD. Menopause. Lancet. 371:760-70, 2008. http://dx.doi.org/10.1016/S01406736(08)60346-3

6. Teles TO, Pereira KF, Souza VR, Paranaiba JS, Teixeira CS. Consequências do climatério e menopausa na sexualidade: um estudo no centro de atendimento integrado à saúde de Rio Verde-Goiás. Rev. Saúde e Biol., v.7, n.3, p.45-51, set./dez., 2012.

7. Galvão LLLF, Farias MCS, Azevedo PRM, Vilar MJP, Azevedo DG. Prevalência de transtornos mentais comuns e avaliação da qualidade de vida no climatério. Rev Assoc Med Bras. 53(5):414-20, 2007. http://dx.doi.org/10.1590/s0104-

\section{$\underline{42302007000500017}$}

8. Silva AR; Ferreira TF; Tanaka ACA. História ginecológica e sintomatologia climatérica de mulheres pertencentes a uma unidade de saúde pública do estado do Acre. Rev. Bras. Cresc. e Desenv. Hum. 20(3) 778786, 2010.

9. Vallance JK, Murray TC, Johnson ST, Elavsky S. Quality of life and psychosocial health in postmenopausal women achieving public health guidelines for physical activity. Menopause. $\quad$ 17(1):64-71, 2010. http://dx.doi.org/10.1097/gme.0b013e3181b $\underline{6690 c}$

10. Gonçalves AKS, Canário ACG, Cabral PUL, Silva RAH, Spyrides MHC, Giraldo PC, Eleutério Jr. J. Impacto da atividade física na qualidade de vida de mulheres de meia idade: estudo de base populacional. Rev Bras Ginecol Obstet. 33(12):408-13, 2011. http://dx.doi.org/10.1590/S0100$\underline{72032011001200006}$

11. Souza Santos CA, Dantas EE, Moreira $\mathrm{MH}$. Correlation of physical aptitude; functional capacity, corporal balance and quality of life (QoL) among elderly women submitted to a post-menopausal physical activities program. Arch Gerontol Geriatr. 53(3):344-9, 2011.

http://dx.doi.org/10.1016/j.archger.2010.12. $\underline{019}$

12. Kupperman HS, Blatt MHG.

Menopausal indice. J Clin Endocrinol. v. 13, n.1, p. 688-694, 1953.

http://dx.doi.org/10.1210/jcem-13-6-688

13. Ministério da Saúde (BR). Assistência Integral à Saúde da Mulher: bases de ação programática. Brasília (DF); 1984.

14. Silva Filho EA, Costa AM. Avaliação da qualidade de vida de mulheres no climatério atendidas em hospital-escola na cidade do Recife, Brasil. Rev Bras Ginecol Obstet. 30:113-20, 2008.

http://dx.doi.org/10.1590/S0100$\underline{72032008005000001}$

15. Pines $P$, Sturdee $D W$, Martin $M$, Schneider HPG, Gambacciani M, Panay N. Recomendações atualizadas sobre a Terapia Hormonal pós-menopáusica. Sociedade Internacional da Menopausa. Clmateric, v. 10, p.181-194, 2007.

16. Pedro AO, Pinto-Neto AM, CostaPaiva LHS, Osis MJD, Hardy EE. Síndrome do climatério: inquérito populacional domiciliar 
em Campinas, SP. Rev. Saúde Pública, vol.37, no.6, p.735-742, 2003.

http://dx.doi.org/10.1590/S0034-

\section{8}

17. Coelho $M$, Salvado A, Robalo $L$. Incontinência urinária feminina: prevalência em 2006 e factores predisponentes na população de Setúbal. Essfisionline, 3(1): 314, 2007.

18. Dias R, Oliveira AT, Vespasiano BS, Nascimento DC, Prestes J. O treinamento de força melhora os sintomas climatéricos em mulheres sedentárias na pós-menopausa. ConScientiae Saúde, 12(2):249-258, 2013.

19. Valença CN, Germano RM. Concepções de mulheres sobre menopausa e climatério. Rev RENE. 11 (1):161-71, 2010.

20. Santos LM, Eserian PV, Rachid LP, Cacciatore A, Bourget IMM, Rojas AC, et al. Síndrome do climatério e qualidade de vida: uma percepção das mulheres nessa fase da vida. Rev APS. 10(1):20-6, 2007.

Recebido para publicação em 19/08/2014

Revisado em 20/08/2014

Aceito em 25/08/2014 